\title{
19 Stellungnahmen der Patienten zur Krankheit
}

\author{
„Rechts und links vom Grat der Symptomfreiheit aber lauert der Abgrund \\ existentieller Angst“ \\ W-P H
}

\subsection{Vorbemerkungen}

Erstaunlicherweise gibt es wenig Literatur über diese Thematik, so, als ob die Frage, wie junge Menschen ihre Psychose erleben und wie sie mit diesem Einbruch in ihr Leben umgehen, uninteressant sei. Vielleicht hängt das mit einer Abwehr gegen das Leid, die Ängste und die Verzweiflung der Betroffenen zusammen. In jüngerer Zeit hat sich Bürgy (2003) mit dem Phänomen der Verzweiflung bei schizophrenen Erwachsenen beschäftigt. Er beklagt ebenfalls, dass dieser affektive Zustand in der psychiatrischen Literatur kaum Beachtung gefunden habe, obwohl „die Verzweiflung das ganze Dasein des Schizophrenen erfasst, sein Wesen bestimmt und in der Nähe der schizophrenen Crundstörung angesiedelt werden muss“ (Bürgy 2003, S. 1).

\subsection{Reaktionsweisen während der akuten Erkrankung}

Sechs der 57 im Kindesalter erkrankten Patienten der ersten Katamnesestudie (s. Kap 8.3 u. 8.4) hatten während der ersten psychotischen Episode und auch in späteren Krankheitsphasen das Gefühl, verrückt zu werden. Es handelte sich dabei jeweils um Patienten mit akut-rezidivierenden Verläufen, die voll $(n=4)$ oder unter Hinterlassung leichter postpsychotischer Einbußen $(\mathrm{n}=2)$ ausheilten. Bei zwei Patienten 
wiederholte sich diese Angst in verschiedenen psychotischen Phasen. Zahlreiche Patienten reagierten auf den psychotischen Einbruch mit Ratlosigkeit, die Kinder liefen mit ratlos-fragendem Gesichtsausdruck herum, wirkten verstört, hilflos und bisweilen desorientiert. Ein 12-jähriges Mädchen drückte das Unerklärliche und Bedrohliche dessen, was es zu erleben schien, in folgenden Worten aus:

„Ich merk' das, dann kommt irgendwas über mich, ich merk' das, das ist eiskalt und dann denke ich nur, jetzt musst du in Sicherheit sein, dass du keine Gehirnerschütterung kriegst“.

Das unbestimmte und beängstigende Gefühl der Bedrohung wird von dem Kind ins Körperliche hineinprojiziert, ähnlich wie es ein 10-jähriger Junge tat, der unvermittelt im Unterricht fragte, ob seine Pulsadern noch ganz seien, und anschließend fortlief. Dies waren die ersten Anzeichen der beginnenden Psychose, die zugleich ein Licht werfen auf die Art des Betroffenseins und Stellungnehmens dazu.

Andere Kinder reagierten mit Verzweiflung und starken depressiven Verstimmungen. Das kommt in den Worten eines 14-jährigen Mädchens in erschütternder Weise zum Ausdruck: „Mir ist, als könnte ich meine Gedanken nicht mehr zusammenhalten, als wollte der Wahnsinn mich erreichen, wie ist mir bang“. Die Trostlosigkeit, die Traurigkeit, die Sorge, die sich bei einigen Kindern auch in schweren Schuldvorstellungen mit herabgesetztem Selbstwertgefühl und wahnhaften Minderwertigkeitsideen äußerten, hielten oft wochenlang an und traten schon im frühen Alter, teilweise vor dem 1o. Lebensjahr auf. Es scheint, dass psychotisches Erleben als eigenes Versagen interpretiert wird, wie es beispielsweise in der Äußerung eines Mädchens den Eltern gegenüber ganz am Anfang ihrer Psychose zum Ausdruck kommt: „Ich kann nicht schlafen, weil ich euch nicht lieben kann“.

Die zitierten und besprochenen Äußerungen zeigen, dass in der akuten Psychose ein Krankheitsgefühl vorhanden ist. Dagegen war eine dauernde und volle Krankheitseinsicht in dieser Krankheitsphase in keinem Fall nachweisbar. Jede Einsicht setzt eine „kritische Selbstbesinnung“ (Störring) voraus, die den psychotischen Kindern fehlte. Bei einem Mädchen zeigte sich eine Krankheitsahnung, jeweils zu Beginn neuer Schübe, die es nahen fühlte, es fühlte sich dann nicht wohl und wollte in die Klinik!

\subsection{Stellungnahmen zur abgelaufenen akuten Psychose}

Nur von wenigen Patienten liegen Äußerungen unmittelbar nach einer durchgemachten psychotischen Episode vor. Das ist verständlich, da jeder Mensch eine besondere Empfindlichkeit gegenüber solchen erlebnismäßig bedeutungsvollen Ereignissen besitzt, die von der öffentlichen Meinung häufig negativ bewertet werden. Ein 13-jähriges Mädchen sagte nach dem Abklingen einer Psychose: „Ich möchte diesen Zustand nicht noch mal erleben, weil die anderen Kinder nicht auch solche Sachen sehen“.

Mehrere Stellungnahmen zu verschiedenen Schüben ihrer akut-häufig rezidivierenden Psychose liegen von einer bei Erkrankungsbeginn 12-jährigen Patientin vor. Im Alter von 15 Jahren sagte sie unmittelbar nach einer schweren psychotischen Episode: „Fragen Sie bitte nicht danach, ich möchte nicht daran erinnert werden. Das ist wirklich alles vorbei. Ich bin dankbar, dass alles vorbei ist“ - und wenige Tage später: „Ich weiß nicht, 
wie das ist, es ist so ganz anders, ich weiß, ich habe sehr getobt, ich habe so manche Erinnerung. Ich habe es nicht gewollt, es kam einfach über mich, es war alles so unheimlich, alles so traumhaft“.

Eine objektive Stellungnahme gegenüber dem in der psychotischen Phase Durchlebten war also ausgesprochen selten. Nur eine einzige Patientin berichtete 20 Jahre nach Erkrankungsbeginn im Alter von 13 Jahren bei der ersten Nachuntersuchung offen über ihre damalige Erkrankung. Sie machte sogar noch weitere Angaben über das psychotische Bild, unter dem sie damals litt, die über die im Krankenblatt fixierten Feststellungen hinausgingen. So gab sie damals im Alter von nunmehr 33 Jahren spontan an, sie habe zusätzlich zu optischen auch unter akustischen Halluzinationen gelitten. So habe sie Stimmen gehört, die ihr Befehle erteilt haben, z.B. nachts „Geh’ doch runter, geh' runter" und die sie beschimpften - Ausführliche Beschreibung siehe Kapitel 2o, Nr. 4.4.1.2b.

Die Stellungnahmen der übrigen Patienten drückten teilweise ratloses Erstaunen aus, teilweise ängstliche Abwehr, gepaart mit einem Gefühl der Erleichterung. Zuweilen jedoch ließ die Einstellung zur Krankheit bereits eine Nivellierung der Persönlichkeit insbesondere auf affektivem Gebiet erkennen und schlug sich in läppischinadäquaten Äußerungen über die bis dahin durchgemachten Krankheitsphasen nieder, wie beispielsweise bei einer 17-jährigen Patientin, die mit 11 Jahren erkrankt war: „fand ich ganz lustig“.

\subsection{Stellungnahme bei chronischen Psychosen}

Von 9 der 15 Patienten mit chronisch verlaufenden Psychosen liegen Äußerungen über ihre Erkrankung vor, die sie im Verlauf der Psychose oder bei der Nachuntersuchung getan hatten. Bemerkenswert ist, dass sie alle nicht über psychotische Erlebnisse und deren Wirkung auf sie selbst berichteten, sondern über die von ihnen registrierte Persönlichkeitsveränderung, die in ihnen vorgegangen war. Erschütternd ist, wie treffend und ehrlich, ohne jede Beschönigung, diese Schilderungen sind. Die Patienten berichteten, dass sie „innerlich ganz verbrannt“, „innerlich leblos und steif“ seien, dass sie „richtig stumpf“ geworden seien, keine Freude mehr empfinden könnten, dass ihnen „alles egal“ sei, sie sich „nicht mehr hundertprozentig begeistern“ könnten. 15 Jahre nach Psychosebeginn sagte ein nunmehr 25-jähriger Patient, früher sei er „gemütsempfindend“ gewesen, ,jetzt bin ich schon so ausgegloist“.

Zwei andere, ebenfalls stark veränderte Patienten - in einem Fall handelte es sich um eine langsam versandende Dementia simplex, im anderen um eine mit 9 Jahren begonnene Hebephrenie - ließen bei der Nachuntersuchung 9 bzw. 19 Jahre nach Krankheitsbeginn erkennen, dass sie zeitweise unter ihrer Antriebs- und Schwunglosigkeit leiden. Sie litten darunter, sich zu nichts mehr entschließen zu können, an nichts mehr Freude und Interesse zu empfinden, und bekundeten, darüber traurig zu sein, „dass es nicht mehr weitergeht“, sich „nicht mehr zusammenreißen“ zu können oder „,nichts zu tun zuhaben“.

Ähnlich drückte sich eine 18-jährige Patientin 6 Jahre nach Psychosebeginn aus: sie sei beunruhigt, dass sie keine Energie mehr habe, sich vor allem nicht mehr richtig überwinden könne - ,ich leide darunter, aber ich kann nicht richtig Mensch sein. Ich habe keine Energie, um zu Hause zu helfen, um halt was zu arbeiten. Es mangelt einfach an Beherrschung." Über ihre früheren künstlerischen Interessen befragt, sagte sie: 
„Mein Talent hat überhaupt nachgelassen in dieser Zeit, das merke ich ganz genau ... es ist nicht mehr so viel da, es wird alles weniger, ooch, ich bin nicht mehr so schnell begeistert, früher war ich mal Feuer und Flamme für etwas, jetzt bin ich das nicht mehr, jetzt bin ich vernünftiger".

Neben den beschriebenen Äußerungen über bei sich selbst registrierte Wesensänderungen war bei drei ebenfalls persönlichkeitsveränderten und dadurch chronisch stationär behandlungsbedürftigen Patienten so etwas wie ein plötzliches Aufblitzen einer Krankheitseinsicht zu bemerken, die jedoch flüchtig war und nur wenige Sekunden anzuhalten schien. So kam es bei einer Patientin während einer psychotischen Episode 6 Jahre nach Krankheitsbeginn zu einem abrupten Wechsel zwischen wahnhaft-verworrenem und gleich darauf klar geordnetem Gedankengang. Unmittelbar nach der Äußerung: „Der heilige Geist hat mich befruchtet, das ist ja komisch, dass ich das nicht weiß", rief sie aus:

„Mit 13 Jahren fing meine Krankheit an, ich wurde nervös, albern, ich werde ja doch nicht wieder gesund, das fühle ich ganz genau“.

\subsection{Epikritische Betrachtung}

Die Reaktionen auf akute schizophrene Episoden waren bei den betroffenen Kindern ähnlich und von dem Gefühl „verrückt“ zu werden, von Ratlosigkeit, Ängsten, Bedrohtheitsgefühlen, Verzweiflung und starken depressiven Stimmungen geprägt. Schuld- und Versagensgefühle sprechen dafür, dass die jungen Patienten sich selbst für den psychotischen Zusammenbruch verantwortlich machten und darauf mit Gefühlen der Trost- und Hoffnungslosigkeit reagierten. Einige Kinder wollten nicht an das, was sie in der Psychose durchgemacht hatten, erinnert werden, oder sie äußerten ihre Dankbarkeit darüber, „dass wirklich alles vorbei war“. Das Gefühl, dass mit ihnen etwas geschehen ist, was sie nicht oder nur vage benennen oder beschreiben konnten, war gepaart mit ratlosem Erstaunen, Irritation, Angst, aber auch mit Erleichterung und der Hoffnung, dass es „nicht wieder kommt“. Solche Gefühle der Bedrohtheit, des Unheimlichen, der Ratlosigkeit, der Angst vor dem Verrücktwerden und der Verzweiflung sind auch bei Erwachsenen beschrieben worden. Lopez-Ibor (1955) deutete die Furcht vor der drohenden Verrücktheit als Furcht vor einer Auflösung der eigenen Persönlichkeit. Entsprechende Ängste vor drohender „Zerstörung“ oder einem Verlust der Ganzheit sind auch bereits von Kindern unserer Studie geäußert worden.

Die Bedrohung der eigenen Person in der Psychose wird vom Kind offenbar häufig leibnah erlebt. Von daher ergibt sich ein Zugang zum Verständnis für die bereits früh sich zeigende Neigung kindlicher Schizophrener zu Coenaesthopathien(vgl. Kap. 4.2). Andererseits reagiert das Kind auch mit schweren und anhaltenden depressiven Verstimmungen, die durchaus bereits denen Erwachsener an Intensität und Dauer gleichkommen. Die Ansicht von Spiel (1969), dass depressive und Angstzustände nur über kürzere Strecken und nie anhaltend zu beobachten seien, und höhere Ausgestaltungen depressiver Inhalte wie „die Sorge um das Heil der Seele, des Daseins, Schuldgefühle, ja sogar ein herabgesetztes Selbstwertgefühl oder gar wahnhafte Ausgestaltungen dem Kindesalter" fremd seien, ist nach unseren Beobachtungen völlig 
unzutreffend, das Gegenteil ist der Fall! Analoge Äußerungen, wie sie Kierkegaard in seinen Tagebüchern niedergeschrieben hat, waren in erstaunlich ähnlichen Formulierungen, die die gleiche Verzweiflung und Trostlosigkeit ausdrückten, auch bei einigen unserer Patienten in der Präpubertätsphase zu finden.

Der Gedanke von Bürgy (2003), dass die Verzweiflung auf ein „fundamentales Fremdwerden des Schizophrenen vor sich selbst und vor dem anderen“ verweise, ist gut nachvollziehbar. Auch die betroffenen Kinder haben zum Ausdruck gebracht, dass sie sich „fremd“, „komisch“ und „verändert" vorkamen. Erstaunlich die Formulierung eines im Alter von 12 Jahren erkrankten Mädchens nach einem schweren psychotischen Rezidiv mit 17 Jahren: „Ich kann gar nicht sagen, die Sache mit dem veränderten Ich hat mich ganz fertig gemacht. Es war alles so komisch, so verändert".

Bei Jugendlichen und jungen Erwachsenen beschrieb Blankenburg (1971) den „Verlust der Selbstverständlichkeit“. Der selbstverständliche Umgang mit den Dingen und Notwendigkeiten der Alltagswelt geht verloren, was sich in den Gefühlen der Fremdheit, Rat- und Orientierungslosigkeit äußert. So fragte ein im Alter von 8 Jahren erkranktes Mädchen der Essener Klinik immer wieder in stereotyper Weise und mit monotoner Stimme „Richtig?“, „Richtig?“. Ähnlich der in Kapitel 17 ausführlich erörterte 19-jährige Jugendliche: „Was ist Leben?“, „Was ist Selbständigkeit?“

Hinter solchen Fragen verbirgt sich der für schizophrene Kinder und Jugendliche so unheimliche und bedrohliche Zusammenbruch des Realitätsbezugs, des Grundvertrauens in eine Sicherheit garantierende Ordnung der Dinge und der Bezüge zur personalen Mitwelt. Das iterative Rückfragen „Richtig, richtig?“ oder „Was ist Leben?“, „Was ist Selbstständigkeit?“ kann als ständige Suche nach Vertrauensbeweisen und als immer wieder von neuem unternommene Versuche des psychotischen Ich verstanden werden, die „Bezüge zur Welt wieder herzustellen und zurecht zu rücken“ (Bürgy 2003).

Der in Kapitel 4.4 (Zwangssymptome) beschriebene Patient (Erkrankungsalter: 11 Jahre) versuchte durch Bannsprüche und Beschwörungsrituale dem drohenden Zerfall seiner Weltordnung entgegen zu wirken. Ein anderer, im Alter von 14 Jahren an einer schleichend beginnenden Psychose erkrankter Junge, stellte, vergleichbar mit den zuvor erwähnten Patienten, stereotyp die gleichen Fragen („Gibt es das, dass kräftige Männer ohne Bewegung dick werden?“, „Schwitzt man viel, wenn man kräftig ist?“, „Muss man sich jeden Tag das Genitale waschen?") um seiner Ratlosigkeit Herr zu werden. Bei der ersten Nachuntersuchung im Alter von 29 Jahren (15 Jahre nach Erkrankungsbeginn) sagte er mir:

„Ich komme manchmal an den Rand, in eine seelische Lage, wo ich aufpassen muss, wo ich die Beherrschung verliere, ich komme dann in eine Art Dämmerzustand, wo ich nicht mehr klar denken kann“.

Er glaube dann, er sei „bescheuert“, und er habe die Gewissheit, es lohne sich nicht weiterzuleben, er habe dann Selbstmordabsichten und leide unter starken Angstzuständen. Er kenne das Gefühl der „Weltuntergangsstimmung“ und der „völligen Ratlosigkeit“.

Bei chronisch-progredient verlaufenden schizophrenen Früherkrankungen stehen Klagen über die psychosebedingten Wesens- und Persönlichkeitsveränderungen, die Reduktion von emotionaler Tiefe und den Verlust von energetischer Dynamik, von Antrieb, Schwung, Motivation und des Interesses an Dingen und Zielen im Vordergrund der Reaktionen auf die psychotische Erkrankung. 
Eine echte Krankheitseinsicht in und nach psychotischen Episoden ist bei kindlichen wie bei Erwachsenen-Schizophrenien äußerst selten (Schulte 1958). M. Müller (1960) sieht gerade in einer unechten Krankheitseinsicht sowie in einer mangelhaften Korrektur etwa noch bestehender Wahnsymptome durch die Patienten und in deren Unfähigkeit, über die durchgemachte Krankheitsphase zu sprechen, eine „Eigenschaft der typischen klassischen Schizophrenien".

W. Schulte (1958), der in Gütersloh ein beeindruckend enges und verständnisvolles Verhältnis zu den Patienten der großen Klinik hatte - er kannte alle Patienten bei ihrem Namen! - schrieb vom „ahnungsvollen Aufleuchten“ einer Krankheitseinsicht „ganz im Beginn der Psychose, offenbar begleitet von dem unheimlichen Gefühl drohender Verwirrung, Selbstaufgabe, Zerstückelung des Ich oder wie immer auch dieses Unausdrückbare angedeutet wird. Solche Kranke werden mit den vielen neuartigen Eindrücken, denen sie ausgesetzt sind, nicht fertig, fragen nach dem Sinn und dem Warum. Sie lassen u.U. schon einen ganz eigentümlichen Blick des Grauens erkennen." (Schulte 1958)

Einige der von uns nachuntersuchten Patienten, die ihre Erkrankung auf eine „Nervenschwäche“, „Nervenzusammenbrüche“, „Überarbeitung“ oder starke Erschöpfung durch innere Konflikte oder äußere Belastungen zurückführten, bestätigen die Ansicht von Schulte (1963), dass in diesen Bemühungen Schizophrener, persönlichkeitsfremde Belastungen vorzuschützen, eine „Art Schutz vor der Vergegenwärtigung endogenen Krankseins, aber auch Sicherung gegenüber der Furcht vor zukünftiger Wiederholung " $z$ u sehen sei.

Im Gegensatz zu der fehlenden Bereitschaft, über psychotische Erlebnisse zu sprechen und sie zu bewerten, scheinen sich manche, insbesondere intelligente Patienten der sich vollziehenden Umstrukturierung ihres Selbst irgendwie bewusst zu werden, und sie äußern sich auch freimütig darüber. Manche von ihnen, wie das zitierte 18-jährige Mädchen, scheinen die in ihnen vor sich gehende Wesensänderung als angenehm zu empfinden, sie gelangten teilweise $z u$ ähnlich befriedigten Äußerungen über sich selbst (vgl. Kap. 19.4), wie es der vordem scheue, in sich gekehrte und an sich und der Welt leidende Hölderlin später im Verlauf seiner schizophrenen Erkrankung mit folgenden Worten beschreibt: „... darfich wohl sagen, dass ich fester und sicherer geworden bin“" (zitiert nach Jaspers 1949).

Gerade auch sog. „Defekt-Schizophrene“ leiden, wie sich in ihren Stellungnahmen zum eigenen Kranksein zeigt. Auch sie sind suizidgefährdet (vgl. Kap. 10). Auch sie leiden - im Gegensatz zur Ansicht von Tölle - ähnlich wie Depressive darunter, nicht mehr traurig sein und sich nicht mehr freuen zu können, also unter dem Gefühl, welches nach Schulte den Kern melancholischen Erlebens ausmacht. Wenn auf die Ähnlichkeit der Depressivität von Schizophrenen und Melancholikern verwiesen wird, so muss jedoch dabei bedacht werden, dass das aktuelle Nicht-Mehr-Traurigsein-Können beim Depressiven im allgemeinen auf die akute depressive Phase beschränkt ist, die strukturelle Fähigkeit zur Trauer jedoch erhalten bleibt, während sie beim Schizophrenen oft vermindert ist oder gar ganz verschwindet. Gerade darunter leiden viele Schizophrene. Oft zeigte sich dies in Form ungelenker und hilflos wirkender kurzer Äußerungen. Dies darf jedoch nicht darüber hinwegtäuschen, dass auch der persönlichkeitsveränderte Schizophrene zu vielen „Regungen feinsinniger Art des Taktes" (Schulte 1958), der Empfindsamkeit und Anhänglichkeit fähig ist, manchmal „zarter und wahrer, wenn auch spröder als der so gut funktionierende, schablonisierte, an Konventionen gebundene Gesunde“ (Schulte 1963). Bei den beiden Nach- 
untersuchungen konnte die Erfahrung bestätigt werden, die Wyrsch (1966) bei seinen schizophrenen Patienten machte, dass im Gespräch mit ihnen das „so oft beschriebene Symptom des sog. schlechten affektiven Rapports oder der Gefühlssteifigkeit“ verschwand.

Beim Versuch, die Einstellung zur Psychose beim schizophrenen Kind und später beim schizophrenen Jugendlichen und schließlich Erwachsenen in den verschiedenen Stadien der Erkrankung zu verstehen, ergeben sich auch wunderbare Einblicke in das Wesen und in die Persönlichkeitsartung des Kranken selbst. Begriffe zu deren Differenzierung und Kennzeichnung, wie etwa „Defekt“ oder „Persönlichkeitsabwandlung“, haben sich in ihrer oft schlagwortartigen Klischeehaftigkeit als völlig unzureichend erwiesen. Solche Etikettierungen sind vielmehr entwertend und versperren den Zugang zur Vielfalt schizophrener Seins-Möglichkeiten und zur Differenziertheit und Tiefe der Persönlichkeitsstrukturen schizophrener Patienten. Keinesfalls können solche Vokabeln das ganz andere In-der-Welt-Sein des Schizophrenen wiedergeben.

Hier offenbaren sich also - neben den bislang dargestellten, durch die Grenzen des Selbstverständnisses bedingten verschiedenartigen Reaktions- und Verarbeitungsformen und unterschiedlichen Weisen der kritischen Stellungnahme der Kranken zur Psychose - die Grenzen des Fremdverständnisses durch den Arzt sowie die Problematik der adäquaten sprachlichen Formulierung. Die Grenzen der Verstehbarkeit und Definierbarkeit schizophrenen Seins - des Geworden-Seins wie des Noch-Werdens - auf die wir immer wieder stoßen bei der Beschäftigung mit dem Schizophrenen und dessen Krankheitsverlauf, sind stets auch die Grenzen der Begreiflichkeit des Anderen, der uns als Partnerbegegnet:

„Niemand kann in sein partnerisches Du wirklich hineinsehen. Die partnerische, mitmenschliche Ursprünglichkeit in der ihr eigenen Weise zu sein, ist uns, den Begegnenden, entzogen und bleibt das Andere gemäß der Heterologik des Ursprungs. So gesehen ist das Du immer das Andere, immer das Anfängliche und auch immer das Übertreffende gegenüber allen Worten und Gedanken“ (Welte 1969). 